\title{
Association of Neutrophil to Lymphocyte Ratio with Patient Reported Outcomes in Knee Osteoarthritis
}

\author{
Marius Ioniţescu ${ }^{1}$, Dinu Vermeşan ${ }^{1}$, Horia Haraguș ${ }^{1, *}$, Oana Suciu ${ }^{2, *} \mathbb{( D}$, Adrian Todor ${ }^{3}{ }^{(}$, \\ Cristian V. Dumitrașcu ${ }^{1}$, Romeo Negrea ${ }^{4}$, Teodora Hoinoiu ${ }^{5}$ (), Daniela Radu ${ }^{6}$ and Dan Fruja ${ }^{7}$ \\ 1 Department of Orthopedics and Trauma, "Victor Babes" University of Medicine and Pharmacy, No 2 Eftimie \\ Murgu Square, 300041 Timisoara, Romania; mariusionitescu@gmail.com (M.I.); dinu@vermesan.ro (D.V.); \\ d.victorcristian@yahoo.com (C.V.D.) \\ 2 Department of Rehabilitation, "Victor Babes" University of Medicine and Pharmacy, \\ No 2 Eftimie Murgu Square, 300041 Timisoara, Romania \\ 3 Department of Orthopaedics, Traumatology and Paediatric Orthopaedics, "IuliuHatieganu" University of \\ Medicine and Pharmacy, No 8 Victor Babes Str, 400012 Cluj-Napoca, Romania; adrian.todor@umfcluj.ro \\ 4 Department of Mathematics, Polytechnic University of Timisoara, No 2 Victoriei Square, \\ 300041 Timisoara, Romania; romeo.negrea@gmail.com \\ 5 Department of Clinical Practical Skills, "Victor Babes" University of Medicine and Pharmacy, \\ No 2 Eftimie Murgu Square, 300041 Timisoara, Romania; tstoichitoiu@umft.ro \\ 6 Department of Surgery, “Victor Babes" University of Medicine and Pharmacy, No 2 Eftimie Murgu Square, \\ 300041 Timisoara, Romania; daniela_radu@hotmail.com \\ 7 Faculty of Medicine, West University "Vasile Goldiş", No 86 Liviu Rebreanu Str, 310025 Arad, Romania; \\ frujad@gmail.com \\ * Correspondence: horia.haragus@umft.ro (H.H.); oanasuciu78@umft.ro (O.S.)
}

Received: 12 October 2020; Accepted: 13 November 2020; Published: 18 November 2020

\begin{abstract}
Background: Determining the association of neutrophil to lymphocyte ratio (NLR) and clinical disease severity in patients with mild and advanced stage knee osteoarthritis (OA).Method: We analyzed clinical scores and routine hematology panels of 111 subjects with symptomatic knee degeneration: mild OA (66) who underwent knee arthroscopy and advanced OA (45) who had total replacement. Results: Compared to the advanced OA group, the mild OA group was younger (54/67), had fewer females (1.75/5.43), and better scores: Euroqol EQ5D Index (0.50/0.25), VAS (visual analog scale) (65/44); International knee documentation committee subjective knee evaluation form-IKDC (31.6/20.24); Knee disability and osteoarthritis outcome score for joint replacement-KOOSJR (15.5/18.3); and better Kellgren-Lawrence stage (1.3/3.8). Patients with advanced disease had higher NLRs compared to mild OA group and controls: 2.82 versus 1.99 $(p=0.004)$ and $1.98(p=0.002)$. In the multiple regression model, NLR was only influenced by age $(p<0.001)$. In the subgroup analysis, for the early OA cases, NLR was significantly dependent to VAS $(p=0.006)$, IKDC $(p=0.001)$ and KOOSJR $(p<0.001)$. Conclusion: NLR was not associated with symptomatic knee $\mathrm{OA}$, as determined by commonly used patient reported outcomes. However, for patients with mild degenerative modifications, EQ5D, VAS, and IKDC were independent predictors of NLR.
\end{abstract}

Keywords: knee joint; osteoarthritis; inflammation mediators; neutrophil to lymphocyte ratio; patient reported outcome measures; arthroscopy; arthroplasty

\section{Introduction}

Knee degeneration covers a wide range of symptoms starting in adult life and progressing with age. It has a high prevalence and is a leading cause of pain and disability [1]. Diagnosis of knee 
osteoarthritis (OA) is mainly based on clinical symptoms. Different measures and treatments apply, according to age and disease severity, including patient reported outcomes and imaging studies $[2,3]$.

Inflammatory mechanisms play an important role in the pathogenesis of OA, expressing changes to cytokines, microRNAs, and metabolites $[1,4,5]$. Several synovial cytokines (IL-10, IL-12, IL-13, SCGF- $\beta$, VEGF) correlate with knee pain and function [6].

Neutrophil to lymphocyte ratio (NLR) is a simple, readily available marker of general inflammation. It has been successfully used to predict malignancy and cardiovascular outcomes [7-9]. More recently, it has been proposed as an independent factor to predict hip and knee radiographic severity, as well as modulate the postoperative period after arthroplasty [10-14]. However, none of these studies examined the association between NLR and commonly used patient reported outcomes (PROs) for knee OA.

Therefore, the main purpose of our study was to determine if there is any association between NLR and clinical disease severity (measured by PROs) in patients with early and advanced stage knee OA.

\section{Materials and Methods}

We included 111 consecutive subjects who were examined in our University affiliated, emergency regional hospital during 2018. Patients with known inflammatory disease, infection, malignancy, acute trauma and incomplete data were not included. We recorded clinical scores and routine hematology panels. Euroqol EQ-5D-5L Index (converted using the UK tariff) and visual analog scale (VAS) were used to determine the general health status $[2,3,15]$. For the NLR study, the laboratory values were retrieved from the hospital's electronic records. The hematology panel at admittance was performed using Nihon Kohden Celltac 6500, Sysmex XT-4000i or ADVIA 2120 analyzer.NLR measurements were taken from peripheral venous blood from elbow plicae punctures in the morning after fasting. The patients were divided in two groups: With mild OA (66) and advanced OA (45).

The mild OA subgroup ( 66 subjects, out of 82 screened), included patients with atraumatic knee pain who underwent arthroscopy according to current clinical practice guidelines [16]. Early OA was defined based on clinical symptoms, MRI and/or arthroscopy operative reports according to criteria recommended by Emery et al. [3]. The subjects completed the international knee documentation committee subjective knee evaluation form (IKDC) and knee disability and osteoarthritis outcome score for joint replacement (KOOSJR). Afterwards the examining physician (surgeon) completed the Tegner-Lysholm knee scoring scale [2,3,15,17].

The advanced OA subgroup (45 subjects, out of 73 screened), included patients scheduled for total knee replacement. Diagnosis was made using the ACR (American College of Rheumatology) or EULAR (European League Against Rheumatism) criteria for knee osteoarthritis [1]. Indication for arthroplasty was made based on clinical and radiographic criteria. The subjects completed IKDC and KOOSJR. The treating physician (surgeon) completed the two parts of the original (1989) version of the knee society score (KSS1-part1 knee score and KSS2-part 2 function) $[2,3,15,17]$. Both pre and postoperative NLR valid values were available and included in the analysis.

A control group of asymptomatic subjects $(n=1260)$ for NLR was evaluated by retrieving medical records of hospital employees' medical visits over the last couple of years. We used the backward selection procedure to optimize a multiple linear regression model with respond (dependent) variable NLR, for all sets of data using $\mathrm{R}$ version 3.5.3 statistical software [18]. Predictor variables were age, gender, Index, VAS, Tegner-Lysholm, IKDC, KOOSJR, KSS1, KSS2, Neutrophil and Lymphocyte values. The statistical decisions were based on $t$-test, F-test, (multiple) coefficient of determination ( $R^{2}-$ Multiple $\mathrm{R}$-squared) and adjusted coefficient of determination $\left(\mathrm{R}_{\mathrm{a}}{ }_{\mathrm{a}}\right.$-Adjusted $\mathrm{R}$-squared). The study was conducted in accordance with the Declaration of Helsinki and the protocol was approved by the Emergency clinical county hospital 'Pius Brinzeu' Timisoara 'Local ethics committee for scientific research', no 176 from 18 October2019. All patients gave their verbal informed consent for inclusion before they participated in the study. 


\section{Results}

There were differences in demographics and NLR between the OA patients (111) and healthy controls: age 59.3 versus 42.4 years old $(p<0.001)$; M:F ratio 1:2.58 versus 1:5.2 $(p<0.001)$; average NLR 2.33 versus $1.98(p=0.007)$. Subgroup analysis between the mild and advanced OA cases is presented in Table 1, Figure 1. Medium values obtained are analyzed in comparison with the normal values from literature. EuroQol EQ5D5L Index normal values-using the UK tariff range - 0.285 to 1 , with negative values for health states worse than death, 0 deaths and 1 best health state possible [16]. VAS normal values were considered in the range 0-100, with 100 representing best health stateimaginable [15]. IKDC (International Knee Documentation Committee—subjective knee form): range 0-100, with 100 representing no impairment [15]; KOOSJR (Knee disability and Osteoarthritis Outcome Score for Joint Replacement): raw score range 0-28, with 28 representing total knee disability [14]; Neutrophil to lymphocyte ratio (NLR): normal mean value 1.65 (1.96 SD: 0.78-3.53) (95\% CI [0.75-0.81] and [3.40-3.66]) [19]. Patients with more advanced disease had higher NLRs. The NLR of the mild OA group was comparable to that of the controls $(p=0.875)$, whereas the advanced OA group NLR was significantly higher $(p=0.002)$.

Table 1. Demographics and clinical disease severity between the mild and severe OA groups.

\begin{tabular}{cccc}
\hline & Mild OA (n = 66) & Advanced OA (n= 45) & $p$ \\
\hline NLR & 1.999 & 2.821 & 0.004 \\
Age & 54.06 & 67.11 & $<0.001$ \\
M:F ratio & $1: 1.75$ & $1: 5.43$ & 0.011 \\
Index & 0.505 & 0.253 & $<0.001$ \\
VAS & 64.92 & 43.88 & $<0.001$ \\
IKDC & 31.66 & 20.24 & $<0.001$ \\
KOOSJR & 1.45 & 18.33 & $<0.001$ \\
Kellgren-Lawrence & 1.3 & 3.8 & $<0.001$ \\
\hline
\end{tabular}

Subgroup analysis of the patients with early versus advanced OA: n: number of subjects; Index: higher values indicate better health; VAS: higher values indicate better health; IKDC: higher values indicate better health; KOOSJR: lower values indicate better health.

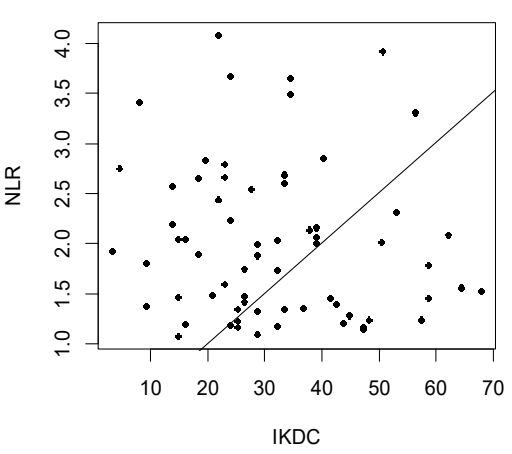

(a)

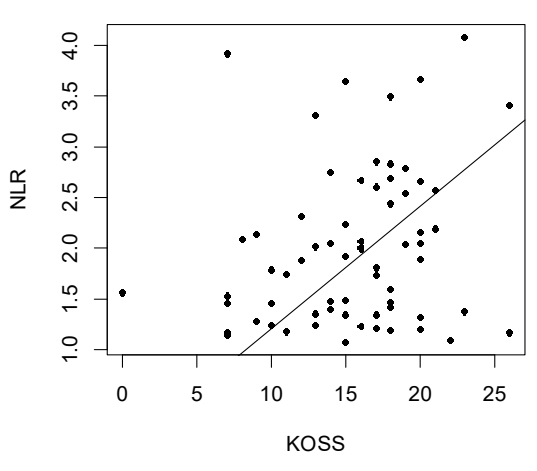

(b)

Figure 1. (a) Mild OA subgroup linear regression IKDC versus NLR. (b) Mild OA subgroup analysis linear regression KOOSJR versus NLR.

In the multiple regression model, NLR of patients with mild and advanced knee degeneration was only influenced by age (Table 2). 
Table 2. Multiple regression for the entire OA group ( $p$-value F-test $<0.001$, multiple coefficient of determination 0.9505 , adjusted coefficient of determination 0.9467$)$ and for the mild OA group (( $p$-value F-test $<0.001$, multiple coefficient of determination 0.9839, Adjusted coefficient of determination 0.9814).

\begin{tabular}{cccc}
\hline $\mathbf{n}=\mathbf{1 1 1}$ & $\boldsymbol{p}$ & $\mathbf{n}=\mathbf{6 6}$ & $\boldsymbol{p}$ \\
\hline Age & $<0.001$ & Age & 0.133 \\
Gender & 0.362 & Gender & 0.610 \\
Index & 0.673 & Index & 0.331 \\
VAS & 0.287 & VAS & 0.006 \\
IKDC & 0.091 & IKDC & 0.001 \\
KOOSJR & 0.123 & KOOSJR & $<0.001$ \\
Neutrophil & $<0.001$ & Neutrophil & $<0.001$ \\
Lymphocyte & $<0.001$ & Lymphocyte & $<0.001$ \\
\hline
\end{tabular}

We studied the homoscedasticity (using the Bartlett test) and the normality (using the Shapiro-Wilk test). We obtained some bad results. The Bartlett test (values from the R soft are: Bartlett's K-squared $=9.0238, d f=2, p$-value $=0.01098)$, results which rejects the null hypothesis of homoscedasticity. For the Shapiro-Wilk test $\left(\mathrm{W}=0.90245, p\right.$-value $\left.=7.79 \times 10^{-5}\right)$ we found the same decision, i.e., reject the hypothesis of normality. These results are based on the high variability of nonoperated patients.

In the subgroup analysis, for the mild OA cases, NLR was significantly dependent to VAS, IKDC and KOOSJR (Table 2). For the advanced disease cases, NLR was only influenced by age (Table 3, Figure 2).

Table 3. Multiple regression for the advanced OA group. Multiple linear regression model subgroup analysis for the advanced OA group: We analyzed two sets of NRLs: NRLpre (before surgery) and NRLpost (the first day after surgery) $p$-value F-test $<0.001$, multiple coefficient of determination 0.9893 , Adjusted coefficient of determination 0.9854 .

\begin{tabular}{cccc}
\hline $\mathbf{n}=\mathbf{4 5}$ & $\boldsymbol{p}$ & $\mathbf{n}=\mathbf{4 5}$ & $\boldsymbol{p}$ \\
\hline Age & 0.001 & Age & $<0.001$ \\
Gender & 0.808 & Gender & 0.725 \\
Index & 0.924 & Index & 0.171 \\
VAS & 0.913 & VAS & 0.092 \\
KSS1 & 0.492 & KSS1 & 0.120 \\
KSS2 & 0.290 & KSS2 & 0.074 \\
IKDC & 0.486 & IKDC & 0.062 \\
KOOSJR & 0.799 & KOOSJR & 0.693 \\
Neutro_pre & $<0.001$ & Neutro_pre & 0.629 \\
Lympho_pre & $<0.001$ & Lympho_pre & 0.643 \\
Neutro_post & 0.023 & Neutro_post & $<0.001$ \\
Lympho_post & 0.595 & Lympho_post & $<0.001$ \\
\hline
\end{tabular}

We also studied the homoscedasticity of the advanced OA group (using the Bartlett test) and the normality (using Shapiro-Wilk test). We obtained good result for this group. The Bartlett test (with the following results from R software: Bartlett's K-squared $=0.054705, d f=2, p$-value $=0.973$ ) which states that we cannot reject the null hypothesis of homoscedasticity. The Shapiro test ( $\mathrm{W}=0.97344$, $p$-value $=0.3834)$ suggests that we cannot reject the normal distribution. These good results for both tests are due to the fact that after the operation the values are strongly comparable. 


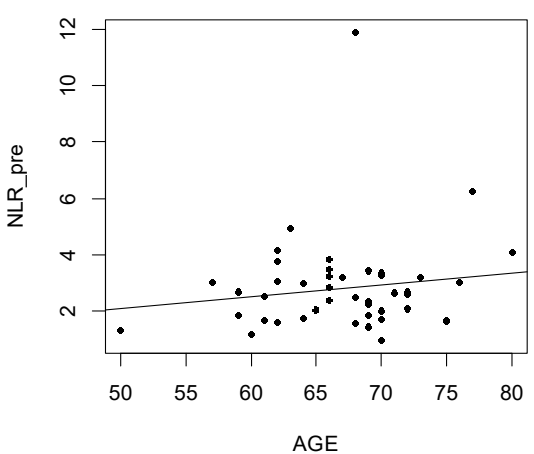

(a)

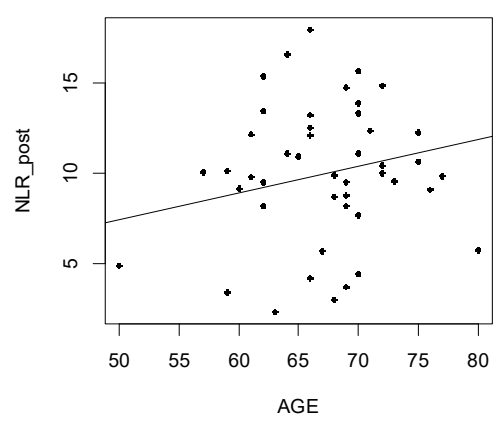

(b)

Figure 2. (a) Advanced OA subgroup analysis linear regression Age versus NLR preop; (b) Advanced OA subgroup analysis linear regression Age versus NLR postop.

\section{Discussion}

Our study is the first to analyze the association of NLR to clinical symptoms in mild and severe knee OA, as determined by PROs. The main finding of our study is that NLR was not associated with disease severity as determined by commonly used PROs. In the subgroup multivariate analysis, for patients with mild degenerative modifications, EQ5D VAS, IKDC and KOOSJR were independent predictors of NLR. In addition, age was significantly associated with NLR values for both symptomatic patients as well as healthy controls. Previous research divided patients based on Kellgren-Lawrence radiographic classification into 2 groups: Grades 1 to 3 (mild to moderate) group and grade 4 another group. Age and NLR>2.1 were independent predictors of advanced OA. By comparison, our patients had higher average NLR values: $1.99 / 2.82$ versus $1.79 / 2.18$ and 1.91/2.16 respectively $[10,11]$. Although not great, these differences appear prominent in the severe OA cases. The difference may be explained by the fact that our advanced OA cases were analyzed just before total knee replacement surgery and therefore, possibly, in a more symptomatic time point.

Knee degeneration has a broad spectrum, from early modifications to advanced disease. Due to our low number of patients (66) we could not compute the association of NLR to cartilage degeneration, meniscus tears and synovitis. We did not include radiographic Kellgren-Lawrence grading in the numerical analysis because, in our opinion, it would not have been relevant for the early stages. It has been shown that patients with early forms of knee OA have significant overlap of symptom severity with cases in end-stage radiographic aspect $[19,20]$. We felt that using common, validated PROs may provide a wider view of symptom severity $[14,17,21]$. Although not perfect, a combination of standardized general and disease specific scores, as well as patient completed and clinician evaluation is more appropriate in defining knee OA burden $[2,3,15,17]$.

Forget et al., determined that normal NLR value in healthy adults should be considered 1.65 but within a broader interval 0.78-3.53 [22]. However, the average value for NLR has also been reported to be higher (2.15) and it is race dependent [23]. In our study, the control group had an average NLR of 1.98 with a higher proportion of females.

There is a plethora of inflammation biomarkers studied in knee OA, mainly produced by synoviocites and chondrocytes and released into the synovial fluid [6]. The most important are pro-inflammatory cytokines interleukin-1 (IL-1), IL-6, IL-8 and tumor necrosis factor- $\alpha$ (TNF- $\alpha$ ), as well as fibroblast growth factor 2 (FGF2), transforming growth factor $\beta$ (TGF- $\beta$ ) and matrix metalloproteinases $[4,5,10,11]$. In our study, we only analyzed NLR because it is readily available, the rest of osteoarthritis markers being of little routine, clinical value and expensive.

NLR as well as platelet to lymphocyte ratio and mean platelet volume are linked to inflammatory arthritis activity such as rheumatoid arthritis, ankylosing spondylitis, psoriasis, or lupus [12]. Rheumatoid arthritis is the prototype inflammatory autoimmune diseases with joint destruction. 
The dynamic evolution of synovial inflammation and excessive CD4+ T-cell mediated immune response can be followed up using NLR. It has comparable efficacy to CRP (C reactive protein) and ESR (erythrocyte sedimentation rate) but is less influenced by cytokines [5,24]. Patients with fractures and trauma have higher NLR compared to controls. However, neither age, gender nor admission NLR or CRP are useful in predicting short term postoperative mortality in elderly patients with hip fracture [25].

A significant limitation is our low number of subjects and somewhat bimodal distribution of disease severity (early and advanced). In addition, we did not analyze the relationship between preoperative PROs and NLR values. We also did not analyze the medium- or long-term outcomes or adverse events. Post treatment evaluation was only available for patients who underwent total knee replacement (advanced OA, $n=45$ ) and confined to immediately postoperative NLR. Furthermore, we did not account for the body mass index. Apart from age and gender (worse for female), obesity has been shown to aggravate pain intensity and function in patients with knee OA [1,5]. Early diagnosis and interventions are important due to financial burden of surgical interventions in osteoarthritis and other pathologies [26,27].

\section{Conclusions}

Neutrophil to lymphocyte ratio was not associated with symptomatic knee OA, as determined by commonly used PROs. However, for patients with mild degenerative modifications, EQ5D VAS, IKDC subjective form and KOOSJR were independent predictors of NLR. A larger study group could verify these results.

Author Contributions: Conceptualization, M.I., O.S., D.F. and H.H.; methodology, M.I., D.V., A.T., C.V.D., T.H., D.R.; software, H.H. and R.N.; validation, M.I., H.H., O.S., A.T., R.N. and D.F.; formal analysis, M.I., O.S., H.H., D.F.; investigation, M.I., D.V., A.T., C.V.D., T.H., R.N., D.R.; data curation, M.I., D.V., A.T., C.V.D., T.H., D.R.; writing —original draft preparation, M.I., O.S., D.F. and H.H.; writing—review and editing, M.I., O.S., D.F. and H.H.; visualization, M.I., D.V., A.T., C.V.D., T.H., D.R., R.N., H.H., O.S. and D.F.; supervision, M.I., O.S., D.F., D.V., D.R., A.T. and H.H. All authors have read and agreed to the published version of the manuscript.

Funding: This research received no external funding.

Acknowledgments: Andrei Ghiorghitoiu.

Conflicts of Interest: The authors declare no conflict of interest.

\section{References}

1. Hunter, D.J.; Bierma-Zeinstra, S. Osteoarthritis. Lancet 2019, 393, 1745-1759. [CrossRef]

2. Wilson, I.; Bohm, E.; Lübbeke, A.; Lyman, S.; Overgaard, S.; Rolfson, O.; W-Dahl, A.; Wilkinson, M.; Dunbar, M. Orthopaedic registries with patient-reported outcome measures. EFORT Open Rev. 2019, 4, 357-367. [CrossRef] [PubMed]

3. Emery, C.A.; Whittaker, J.L.; Mahmoudian, A.; Lohmander, L.S.; Roos, E.M.; Bennell, K.L.; Toomey, C.M.; Reimer, R.A.; Thompson, D.; Ronsky, J.L.; et al. Establishing outcome measures in early knee osteoarthritis. Nat. Rev. Rheumatol. 2019, 15, 438-448. [CrossRef] [PubMed]

4. Boehme, K.A.; Rolauffs, B. Onset and Progression of Human Osteoarthritis-Can Growth Factors, Inflammatory Cytokines, or Differential miRNA Expression Concomitantly Induce Proliferation, ECM Degradation, and Inflammation in Articular Cartilage? Int. J. Mol. Sci. 2018, 19, 2282. [CrossRef] [PubMed]

5. Tateiwa, D.; Yoshikawa, H.; Kaito, T. Cartilage and Bone Destruction in Arthritis: Pathogenesis and Treatment Strategy: A Literature Review. Cells 2019, 8, 818. [CrossRef] [PubMed]

6. Nees, T.A.; Rosshirt, N.; Zhang, J.A.; Reiner, T.; Sorbi, R.; Tripel, E.; Walker, T.; Schiltenwolf, M.; Hagmann, S.; Moradi, B. Synovial Cytokines Significantly Correlate with Osteoarthritis-Related Knee Pain and Disability: Inflammatory Mediators of Potential Clinical Relevance. J. Clin. Med. 2019, 8, 1343. [CrossRef] [PubMed]

7. Wang, D.; Bai, N.; Hu, X.; OuYang, X.W.; Yao, L.; Tao, Y.; Wang, Z. Preoperative inflammatory markers of NLR and PLR as indicators of poor prognosis in resectable HCC. PeerJ 2019, 7, e7132. [CrossRef] 
8. Filh, J.G.M.S.; Torres, D.D.O.C.; Da Silva, M.C.F.C.; Martins, C.M.D.H.; Da Silva, I.K.; Nascimento, M.E.M.D.; Dos Santos, A.C.O.; Montarroyos, U.R.; Filho, D.C.S. Prognostic value of hematological parameters in patients with acute myocardial infarction: Intrahospital outcomes. PLoS ONE 2018, 13, e0194897. [CrossRef]

9. Parvizi, J.; Tan, T.L.; Goswami, K.; Higuera, C.; Della Valle, C.; Chen, A.F.; Shohat, N. The 2018 Definition of Periprosthetic Hip and Knee Infection: An Evidence-Based and Validated Criteria. J. Arthroplast. 2018, 33, 1309-1314.e2. [CrossRef]

10. Taşoğlu, Ö.; Bölük, H.; ŞahinOnat, Ş.; Taşoğlu, İ.; Özgirgin, N. Is blood neutrophil-lymphocyte ratio an independent predictor of knee osteoarthritis severity? Clin. Rheumatol. 2016, 35, 1579-1583. [CrossRef]

11. Büyükavc1, R.; Aktürk, S.; Sağ, S. Comparison of blood platelet distribution width and neutrophil-lymphocyte ratio in patients with different grades of knee osteoarthritis. J. Back Musculoskelet. Rehabil. 2018, 31, 1035-1039. [CrossRef] [PubMed]

12. Taşoğlu, Ö.; Şahin, A.; Karataş, G.; Koyuncu, E.; Taşoğlu, İ.; Tecimel, O.; Özgirgin, N. Blood mean platelet volume and platelet lymphocyte ratio as new predictors of hip osteoarthritis severity. Medicine 2017, 96, e6073. [CrossRef] [PubMed]

13. Yombi, J.C.; Schwab, P.E.; Thienpont, E. Neutrophil-to-lymphocyte ratio (NLR) distribution shows a better kinetic pattern than C-reactive protein distribution for the follow-up of early inflammation after total knee arthroplasty. Knee Surg. Sports Traumatol. Arthrosc. 2016, 24, 3287-3292. [CrossRef] [PubMed]

14. Suciu, O.; Bereteu, L.; Drăgănescu, G. Determination of mechanical properties of hydroxyapatite doped with magnesium. AIP Conf. Proc. 2013, 1564, 132-137.

15. Todor, A.; Vermesan, D.; Haragus, H.; Patrascu, J.M., Jr.; Timar, B.; Cosma, D.I. Cross-cultural adaptation and validation of the Romanian International Knee Documentation Committee-subjective knee form. PeerJ 2020, 8, e8448. [CrossRef]

16. Beaufils, P.; Becker, R.; Kopf, S.; Matthieu, O.; Pujol, N. The knee meniscus: Management of traumatic tears and degenerative lesions. EFORT Open Rev. 2017, 2, 195-203. [CrossRef]

17. Florescu, S.; Vermesan, D.; Haragus, H.; Patrascu, J.M., Jr.; Timar, B.; Todor, A. Cross-cultural adaptation and validation of the Romanian knee disability and osteoarthritis outcome score for joint replacement (KOOSJR). BMC Musculoskelet. Disord. 2020, 21, 155. [CrossRef]

18. R 5.3.5 for Windows. Available online: https://cran.r-project.org/bin/windows/base/old/3.5.3/ (accessed on 12 September 2018).

19. Jones, L.D.; Bottomley, N.; Harris, K.; Jackson, W.; Price, A.J.; Beard, D.J. The clinical symptom profile of early radiographic knee arthritis: A pain and function comparison with advanced disease. Knee Surg. Sports Traumatol. Arthrosc. 2016, 24, 161-168. [CrossRef]

20. Andor, B.; Patrascu, J.; Florescu, S.; Cojocaru, D.; Sandesc, M.; Borcan, F.; Boruga, O.; Bolintineanu, S. Comparison of different knee implants used on patients with osteoarthritis control study. Mater. Plast. 2016, 53, 119-125.

21. Onofrei, R.R.; Amaricai, E.; Petroman, R.; Suciu, O. Relative and absolute within-session reliability of the modified Star Excursion Balance Test in healthy elite athletes. PeerJ 2019, 7, e6999. [CrossRef]

22. Forget, P.; Khalifa, C.; Defour, J.-P.; Latinne, D.; Van Pel, M.-C.; De Kock, M. What is the normal value of the neutrophil-to-lymphocyte ratio? BMC Res. Notes 2017, 10, 1-4. [CrossRef] [PubMed]

23. Azab, B.; Camacho-Rivera, M.; Taioli, E. Average Values and Racial Differences of Neutrophil Lymphocyte Ratio among a Nationally Representative Sample of United States Subjects. PLoS ONE 2014, 9, e112361. [CrossRef] [PubMed]

24. Chandrashekara, S.; Mukhtar Ahmad, M.; Renuka, P.; Anupama, K.R.; Renuka, K. Characterization of neutrophil-to-lymphocyte ratio as a measure of inflammation in rheumatoid arthritis. Int. J. Rheum. Dis. 2017, 20, 1457-1467. [CrossRef] [PubMed]

25. Niessen, R.; Bihin, B.; Gourdin, M.; Yombi, J.-C.; Cornu, O.; Forget, P. Prediction of postoperative mortality in elderly patient with hip fractures: A single-centre, retrospective cohort study. BMC Anesthesiol. 2018, 18, 183. [CrossRef] [PubMed]

26. Boia, E.S.; David, V.L. The Financial Burden of Setting up a Pediatric Robotic Surgery Program. Medicina 2019, 55, 739. [CrossRef] [PubMed] 
27. London, N.J.; Miller, L.E.; Block, J.E. Clinical and economic consequences of the treatment gap in knee osteoarthritis management. Med. Hypotheses 2011, 76, 887-892. [CrossRef] [PubMed]

Publisher's Note: MDPI stays neutral with regard to jurisdictional claims in published maps and institutional affiliations.

(C) 2020 by the authors. Licensee MDPI, Basel, Switzerland. This article is an open access article distributed under the terms and conditions of the Creative Commons Attribution (CC BY) license (http://creativecommons.org/licenses/by/4.0/). 